\title{
The mystery is solved - CatSper is the principal calcium channel activated by progesterone in human spermatozoa
}

\author{
Christopher LR Barratt
}

Asian Journal of Andrology (2011) 13, 351-352; doi:10.1038/aja.2011.9; published online 28 March 2011

A remarkable advance in sperm physiology has recently been published in Nature. Two groups using patch clamping techniques on human sperm have solved a mystery about the sperm cell that has puzzled both andrologists and those involved in nongenomic cellular signalling for over 20 years. In these papers, Lishko ${ }^{1}$ and Strunker ${ }^{2}$ independently demonstrate that the universal characteristic effect of progesterone on sperm-a rapid influx of calcium-is via a sperm-specific channel CatSper.

Undoubtedly, a key breakthrough in these experiments was the routine patch clamping of human spermatozoa, first introduced by Lishko and colleagues. ${ }^{3}$ Although this is a very challenging technique to perform on human cells, it is absolutely necessary in order to provide direct evidence of channel activity and, in this case, stimulation of CatSper with progesterone. The patch clamping data show that the characteristic of the progesteroneinduced channel was identical to that of CatSper. Additional experiments used, for example, specific drugs (NNC 55-0396 and mibefradil) to help identify the channel and, electrophysiological recordings showed that the characteristic of the channel were identical to CatSper, e.g., response to $\mathrm{pH}$. Further experiments by Strunker ${ }^{2}$ and colleagues examined the signalling mechanisms associated with the progesterone response. Progesterone did not elevate cyclic adenosine monophosphate (cAMP) and, manipulation of cAMP (either directly or via inhibition of soluble or membrane adenylcyclase) did not induce a calcium influx. This indicates that cAMP signalling is not involved in the rapid

Reproductive and Developmental Biology, Maternal and Child Health Science Laboratories, Centre for Oncology and Molecular Medicine, Ninewells Hospital, University of Dundee, Dundee, Scotland DD1 9SY, UK Correspondence: Professor CLR Barratt (c.barratt@ dundee.ac.uk) response to progesterone, resolving an outstanding signalling conundrum for sperm biologists.

Perhaps surprisingly the interaction of progesterone with spermatozoa has been known for several decades. In 1989 and 1990, Thomas ${ }^{4}$ and Blackmore ${ }^{5}$ independently documented the rapid, and now characteristic, influx of calcium in human spermatozoa. Subsequently, although a deluge of research papers were published, the identity of the receptor binding progesterone and the channel responsible for the influx remained a mystery. ${ }^{6}$ In fact, prior to the two publications in Nature, ${ }^{1,2}$ our knowledge could be summed up as the calcium response (i) was a result of influx from outside the cells; (ii) was non-genomic (due to the very rapid effect and, that sperm are devoid of an active transcription/translation apparatus); (iii) was blocked by $\mathrm{La}^{3+}$; and (iv) because the response was not blocked by RU486, deemed not to be the classical progesterone receptor. In reality, two decades of subsequent research failed to further these original findings ${ }^{4,5}$ as to the identity of the membrane receptor and channels responsible for the influx of calcium. The primary importance of the two studies ${ }^{1,2}$ is that they have solved a significant and important part of this scientific mystery-the nature of the channel.

So why else are these studies so important? Firstly, clear physiological effects of progesterone have been continually and progressively demonstrated encompassing chemotaxis, hyperactivated motility and, in some cases, either direct stimulation of the acrosome reaction and/or priming the cell to acrosome reaction. ${ }^{6}$ As such we know that human sperm are very sensitive to progesterone and that it produces a number of critical well-documented biological responses in the cell. Of equal importance is that, clinically, there have been a plethora of studies demonstrating that a suboptimal influx of calcium, in response to progesterone, was associated with sperm dysfunction for example reduced fertilisation at in vitro fertilisation. ${ }^{7}$ The response of cells to progesterone is a putative diagnostic test for sperm dysfunction. Thus, there is a wealth of biological and clinical data that can be used as a baseline for further key studies to identify the mechanism involved in important physiological processes such as chemotaxis. Secondly, CatSper is a sperm-specific channel primarily associated with the development of hyperactivation, for example, CatSper $\mathrm{KO}$ mice do not hyperactivate. ${ }^{8}$ This allows specific experiments in humans to explore the relationship between hyperactivation and CatSper. Hyperactivation is strongly associated with fertility in humans and deletions of the CatSper gene have been detected in several men with infertility. ${ }^{9}$ Although currently the link between CatSper and hyperactivation is not absolutely established in human cells, it is very likely and thus we can now study a clinical phenomenon more intelligently to determine if CatSper channel dysfunction is associated with suboptimal hyperactivation. If so, what are the molecular defects? Perhaps, with the design of appropriate drugs modulating CatSper function, we may even be able to treat men who have defects in the CatSper channels. Thirdly, as the calcium response in sperm is a classical example of nongenomic signalling, it will allow detailed investigation of the myriad of systems used in this classical signalling paradigm. In this respect these papers tell us only what is not involved, e.g., cAMP. Fourthly, Lishko and colleagues $^{1}$ identified a clear difference between mice and human spermatozoa in their response to progesterone. Progesterone did not activate CatSper in mice. Differences between human and mouse cells are often overlooked by those working on animals who, usually, wrongly assume that human 
spermatozoa behave in a similar way. They don't. Hyperactivation is a clear illustration. In the human, hyperactivation is very variable and a significant challenge is that the spermatozoa can easily, and rapidly, move from a hyperactivated to a non-hyperactivated state with considerable regularity. ${ }^{10,11}$ While experiments in mice are often interesting, they more often than not have minimal relevance in understanding the fertilisation process in humans. Last but not least, is the real possibility that the CatSper channels represent a target for several different ligands other than progesterone, for example, prostaglandin E.

Interestingly, Strunker et al. ${ }^{2}$ showed that, under capacitating conditions, the affinity of progesterone for the receptor was significantly enhanced. Lishko et al. ${ }^{1}$ also report that capacitation makes CatSper channel more responsive to progesterone by changing its intrinsic property- dependence on membrane voltage. Capacitation is a pivotal part of the fertilisation process involving a mandatory series of ill-defined events (broadly defined as changes that take place in the cell allowing them to be competent to fertilise the egg). While it is necessary to determine if cellular responses change in capacitation conditions, a fundamental challenge is to define the biological, cellular and molecular events associated with capacitation. Almost all experiments, at least in humans, are performed in vitro with little or no relevance to the conditions the cells encounters in vivo. We ignore this discrepancy at our peril. By continuing our experiments divorced from the natural systems, we are likely to be studying the spermatozoa in the wrong environment, in the wrong way and at the wrong time.

All scientific achievements stimulate a plethora of further questions. This is no exception. For example, is CatSper the receptor or is it part of a larger complex? The studies by Lishko ${ }^{1}$ and Strunker ${ }^{2}$ represent a fundamentally important advance and a very strong platform for what is likely to be the genesis of a golden age in sperm research. We will learn fascinating, surprising and perhaps even perplexing information. There is palpable excitement in andrology now that sophisticated tools are available to allow the molecular and cellular basis of sperm dysfunction to be elucidated with much more precision that previously ever imagined possible. ${ }^{12}$ Your local andrologist should be beside themselves with excitement. If not, check he/she has pulse!

1 Lishko PV, Botchkina IL, Kirichok Y. Progesterone activates the Principal $\mathrm{Ca}^{2+}$ channel of human sperm. Nature 2011; 471: 387-91.
2 Strunker T, Goodwin N, Brenker C, Kashikar ND, Weyand I et al. The CatSper channel mediates progesterone-induced $\mathrm{Ca}^{2+}$ influx in human sperm. Nature 2011; 471: 382-86.

3 Lishko PV, Botchkina IL, Fedorenko A, Kirichok Y. Acid extrusion from human spermatozoa is mediated by flagellar voltage-gated proton channel. Cell 2010; 140: 327-37.

4 Thomas P, Meizel S. Phosphatidylinositol 4,5bisphosphate hydrolysis in human sperm stimulated with follicular fluid or progesterone is dependent upon $\mathrm{Ca}^{2+}$ influx. Biochem J 1989; 264: 539-46.

5 Blackmore PF, Beebe SJ, Danforth DR, Alexander N. Progesterone and 17 alpha-hydroxyprogesterone. Novel stimulators of calcium influx in human sperm. J Biol Chem 1990; 265: 1376-80.

6 Publicover S, Harper CV, Barratt C. $\left[\mathrm{Ca}^{2+}\right]_{i}$ signalling in sperm-making the most of what you've got. Nat Cell Biol 2007; 9: 235-42.

7 Krausz C, Bonaccorsi L, Maggio P, Luconi M, Criscuoli $\mathrm{L}$ et al. Two functional assays of sperm responsiveness to progesterone and their predictive values in in-vitro fertilization. Hum Reprod 1996; 11: 1661-7.

8 Chung JJ, Navarro B, Krapivinsky G, Krapivinsky L, Clapham DE. A novel gene required for male fertility and functional CATSPER channel formation in spermatozoa. Nat Commun 2011; 2: 153

9 Hildebrand MS, Avenarius MR, Fellous M, Zhang Y, Meyer NC et al. Genetic male infertility and mutation of CATSPER ion channels. Eur J Hum Genet 2010; 18: 1178-84

10 Pacey AA, Ladbrook MB, Barratt CL, Cooke ID. The potential shortcomings of measuring hyperactivated motility by computer-aided sperm analysis when sperm motion is multiphasic. Hum Reprod Update 1997; 3: 185-93.

11 Mortimer ST. A critical review of the physiological importance and analysis of sperm movement in mammals. Hum Reprod Update 1997; 3: 403-39.

12 Barratt CL, Mansell S, Beaton C, Tardif S, Oxenham SK. Diagnostic tools in male infertility-the question of sperm dysfunction. Asian J Androl 2011; 13: 53-8. 